\title{
A Comparison of Spermiogenesis and Spermatozoal Ultrastructure in Megascolecid and Lumbricid Earthworms (Oligochaeta : Annelida)
}

\author{
B. G. M. Jamieson
}

Department of Zoology, University of Queensland, St. Lucia, Qld 4067.

\begin{abstract}
Spermiogenesis of six species of megascolecid earthworms has been investigated by electron microscopy. Numerous spermatids are joined to a central anucleate mass, forming a morula. Connection is made by a filament-clothed bridge, the zonula collaris. At least nine mitochondria are observed in a young spermatid but in all cases only six persist, with their juxtaposed surfaces radiating from a central axis, in the middle piece. A stack of golgi cisternae is present distally and is associated with the immature acrosome, which is subsequently transported proximally and interposed between nucleus and zonula collaris. Microtubules of the manchette ensheath the acrosome nucleus and incipient middle piece and, subsequently, all cytoplasm peripheral to the manchette with the exception of the plasma membrane is eliminated. Cytomorphogenetic changes, including great elongation and condensation of the nucleus, result in a free filamentous spermatozoon with a tapering tubular acrosome, an extremely elongate nucleus (total length $10 \mu \mathrm{m}$, cf. $20-30 \mu \mathrm{m}$ in Lumbricus) a small but elongate middle piece $(0.5-1.4 \mu \mathrm{m}$ long) and long flagellum. Close agreement thus exists with spermiogenesis in the Lumbricidae, and the acrosome, which is among the most complex in the animal kingdom, shows general homology with that of Lumbricus. Significant differences from Lumbricus include the domed proximal end of the nucleus, the less discrete basal compartment, the conspicuous limen and greater separation of the outer wall of the acrosomal vesicle from the acrosome wall. A centriole with nine satellites peripheral to nine microtubules is demonstrable. A tetragon configuration in which two central fibres are added to the usual nine doublet+two central singlet microtubules is demonstrated in the proximal flagellum after a short region with a single central filament, but further distally the two fibres cease, giving the normal $9+2$. Terminally the doublets are replaced by singlets and the number of microtubules is reduced. Glycogen granules are conspicuous, peripheral to the microtubules through much of the proximal axoneme as in lumbricids. A species of Cryptodrilus shows an asymmetrical $3+2$ arrangement of satellites in a peripheral circlet, which exemplifies the taxonomic value of spermatozoal ultrastructure. It is confirmed that oligochaete spermatozoa are fundamentally different from those of polychaetes and archiannelids.
\end{abstract}

\section{Introduction}

The Megascolecidae is the largest family of the Oligochaeta in numbers of species, and is indigenous in all zoogeographical regions with the exception of the Western Palaearctic, which is the chief focus of the Holarctic family Lumbricidae. Whereas a single species of the Lumbricidae, Lumbricus terrestris Linnaeus, syn. L. herculeus (Savigny), has been the subject of excellent ultrastructure studies of spermiogenesis and spermatozoal anatomy (Gatenby and Dalton 1959; Cameron and Fogal 1963; Anderson et al 1967; Anderson and Ellis 1968; Lanzavecchia and Donin 1972; Henley 1973) no comparable investigation of the Megascolecidae has been made despite the fact that Pheretima is the textbook earthworm type throughout most of Asia and in Australia. The only other published descriptions of spermatozoal ultrastructure in the Oligochaeta are those of Reger (1967) for Enchytraeus albidus and of Ferraguti and Lanzavecchia (1971) for the tubificids Tubifex and Limnodrilus. It is the aim of the 
present study to give a preliminary outline of spermiogenesis and spermatozoal anatomy in the Megascolecidae, represented by five genera and six species of the three constituent tribes of the subfamily Megascolecinae, and to draw comparisons with the above-mentioned accounts of Lumbricus. The six megascolecid species are: Fletcherodrilus unicus Michaelsen, 1891, and Cryptodrilus sp. (Tribe Perionychini); Digaster longmani Boardman, 1932 (Tribe Dichogastrini); Spenceriella sp., Amynthas (= Pheretima) sp. and A. diffringens (Baird, 1869) (Tribe Megascolecini Jamieson, 1971).

\section{Materials and Methods \\ Seminal vesicles and spermathecal diverticula were fixed in cacodylate-buffered glutaraldehyde and post- fixed in phosphate-buffered osmium tetroxide at $4^{\circ} \mathrm{C}$, dehydrated in a graded series of ethanol and in propylene oxide, embedded in Araldite mixture, or transferred from alcohol to Spurr's medium. They were sectioned on an LKB ultramicrotome and photographed on a Siemens 1A electron microscope. All specimens have been lodged in the Queensland Museum.}

\section{Comparative Spermiogenesis}

\section{The Sperm Morula and Spermatids}

Spermatogenesis in earthworms begins in the testes but is completed either in seminal vesicles, as in four of the megascolecid species under investigation, or in testis-sacs, or in both types of receptacle as in Amynthas. The spermatogonia and spermatocytes proliferate synchronously and in association so that groups of 128 or, rarely, 256 spermatids are formed (Bugnion and Popoff 1905; Walsh 1954). Each spermatid of a group is attached to a common central anucleate cytoplasmic mass, the cytophore or blastophore. The group of spermatids, with the anucleate mass, constitutes the sperm morula. Each spermatid transforms without further division into a spermatozoon in the process of spermiogenesis.

A section of a sperm morula of Fletcherodrilus unicus showing the nuclei of some 29 spermatids is seen in Fig. 3. The cytophore, though anucleate, contains numerous small subspherical mitochondria, each with a few cristae, and many groups of ribosomes. Mitochondrial profiles may be several times longer than wide in other preparations of the same species. The spermatid at this early stage is a relatively undifferentiated cell with a large coarsely granular nucleus, a stack of lamellate golgi cisternae (Figs 4, 22) on the opposite side of the nucleus to the attachment to the cytophore, and a number of small mitochrondria. A young spermatid of Digaster examined has nine mitochondria, in two groups, one group lying adjacent to the connection with the cytophore and the other lying more distally relative to the connection. The distal group alone persists, filling the middle piece of the spermatozoon.

The connection of the spermatid to the cytophore is surrounded by a 'fur-like collar' (Gatenby and Dalton 1959, p. 47) or 'fuzzy coat' (Anderson et al 1967, p. 14) shown in longitudinal section in Fig. 5 for Fletcherodrilus and in transverse section in Fig. 18 for Digaster. The term zonula collaris is here proposed for this type of connecting bridge with its fur-like collar.

While the nucleus of the young spermatid is still coarsely granular it becomes invested by longitudinally directed microtubules constituting the manchette. The manchette is seen ensheathing acrosome, nucleus and middle piece in Figs 6 and 7 , and nucleus in Fig. 21. 


\section{The Older Spermatid}

In the older spermatid (Fig. 5, Fletcherodrilus) the cell and its nucleus have greatly elongated and the nuclear contents have become finely granular. The elongate nucleus abuts the zonula collaris, leaving at this stage no room for the acrosome, which will subsequently cap the nucleus. All mitochondria except those which will occupy the middle piece have disappeared and the persistent mitochondria have taken up their definitive position distal to the nucleus, although they have not yet become axially contiguous. The middle piece is not yet partitioned from the incipient sperm head.

At this stage, when the mitochondria are grouping to form the precursor of the middle piece, the flagellum of the tail is well developed. A sheath of granules peripheral to the outer filament of the tail has formed at this stage (e.g. Digaster) or develops later (e.g. Fletcherodrilus). These granules are characteristic of the sperm of Lumbricidae (Anderson et al. 1967, p. 24) and Megascolecidae (six species) and have been shown by those authors to consist of glycogen.

\section{The Centrioles}

There is some confusion in the literature with regard to lumbricid centrioles (Gatenby and Dalton 1959, figs 13, 14, pl. 25, fig. 14; Anderson et al. 1967, pp. 16, 20; Henley 1973).

The existence of a centriole in the mature megascolecid sperm, with relationships to the flagellum similar to those in Lumbricus, is here demonstrated (Fletcherodrilus, Figs 5, 6, 14; Digaster, Fig. 20; Spenceriella) but whether the centriolar apparatus is resolvable into two centrioles in longitudinal sequence, as indicated by Gatenby and Dalton, is questionable. Evidence from Anderson et al. for Lumbricus is against the latter interpretation, for they state (p. 13) that, although there are initially two centrioles, only one persists at the base of the flagellum and the other migrates from the distal pole of the nucleus to the peripheral cytoplasm where it is associated with formation of the acrosome. Even Gatenby and Dalton in their discussion of the acrosome carrier (see below) suggest that this may temporarily contain a centriole which they, somewhat surprisingly, consider the distal centriole.

\section{The Golgi Complex and Acrosome in the Developing Spermatid}

In the six megascolecids studied, as in Lumbricus, the long acrosome is assembled between nucleus and proximal collar (Fig. 7, Fletcherodrilus). In Lumbricus the 'proacrosome' and the 'acrosome carrier', both shown to comprise the acrosome by Anderson and Ellis (1968), arise from the golgi apparatus (acroblast) and move to the tip of the nucleus (Gatenby and Dalton 1959; Cameron and Fogal 1963; Anderson et al. 1967, 1968; Lanzavecchia and Donin 1972). In Fletcherodrilus a structure identifiable as the receptacle-like 'acrosomal carrier' (Gatenby and Dalton) or the dense granule enclosed in dense material within the acrosomal vesicle (Anderson and Ellis 1968) has been observed lying in a nest made by golgi lamellae, much as described and illustrated by those authors (Fig. $4, k$ ). [For an excellent elucidation of the formation of the acrosome, see Lanzavecchia and Donin (1972).] Subsequent location of the developing acrosome in a clavate projection from the cell body, demonstrated by Anderson and Ellis (the 'balloon-like bleb' of Cameron and Fogal), has been observed for Digaster but the mode of transportation of the acrosome in megascolecids still awaits elucidation. There is no reason to believe that it is significantly different from that in Lumbricus, however. Whatever the mode of 


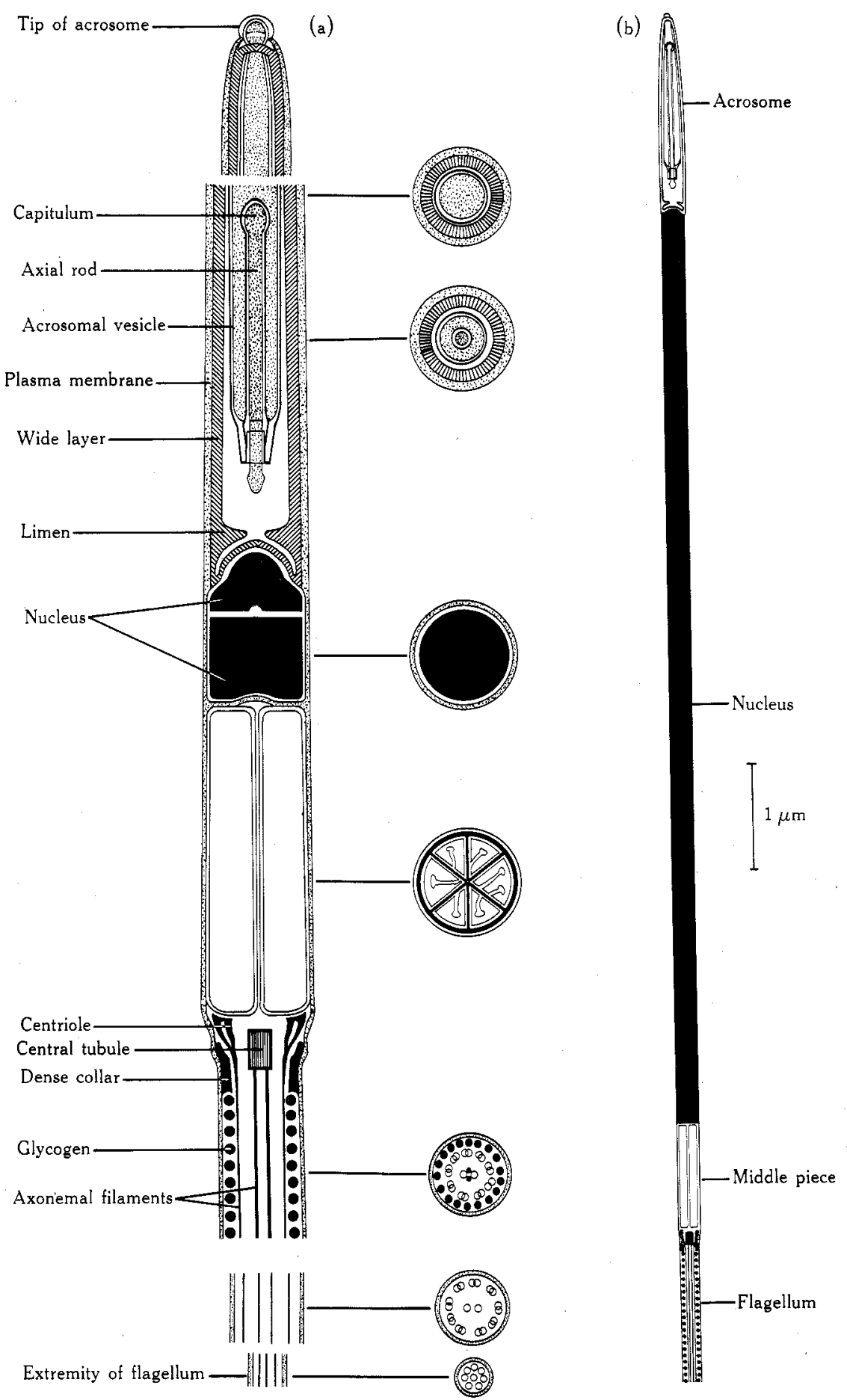

Fig. 1. (a) Diagram of the fine structure of a generalized megascolecid sperm. The structure of the tip of the acrosome and of the centriolar complex requires further elucidation. Transverse sections of various regions are also shown. (b) A spermatozoon of Fletcherodrilus, slightly diagrammatized, to show the relative proportion of acrosome, nucleus and middle piece. Only a short proximal portion of the tail is shown. 
transfer, the relationships between nucleus and its capping acrosome in all six megascolecids shows a constant and significant difference from that in Lumbricus. Cameron and Fogal (1963, p. 757, fig. 1), Anderson and Ellis (1968, figs 7, 10, 11), Lanzavecchia and Donin (1972, fig. 17) and Henley (1973, fig. 4) show that in Lumbricus the acrosome is placed on the flat, or at most slightly convex, anterior end

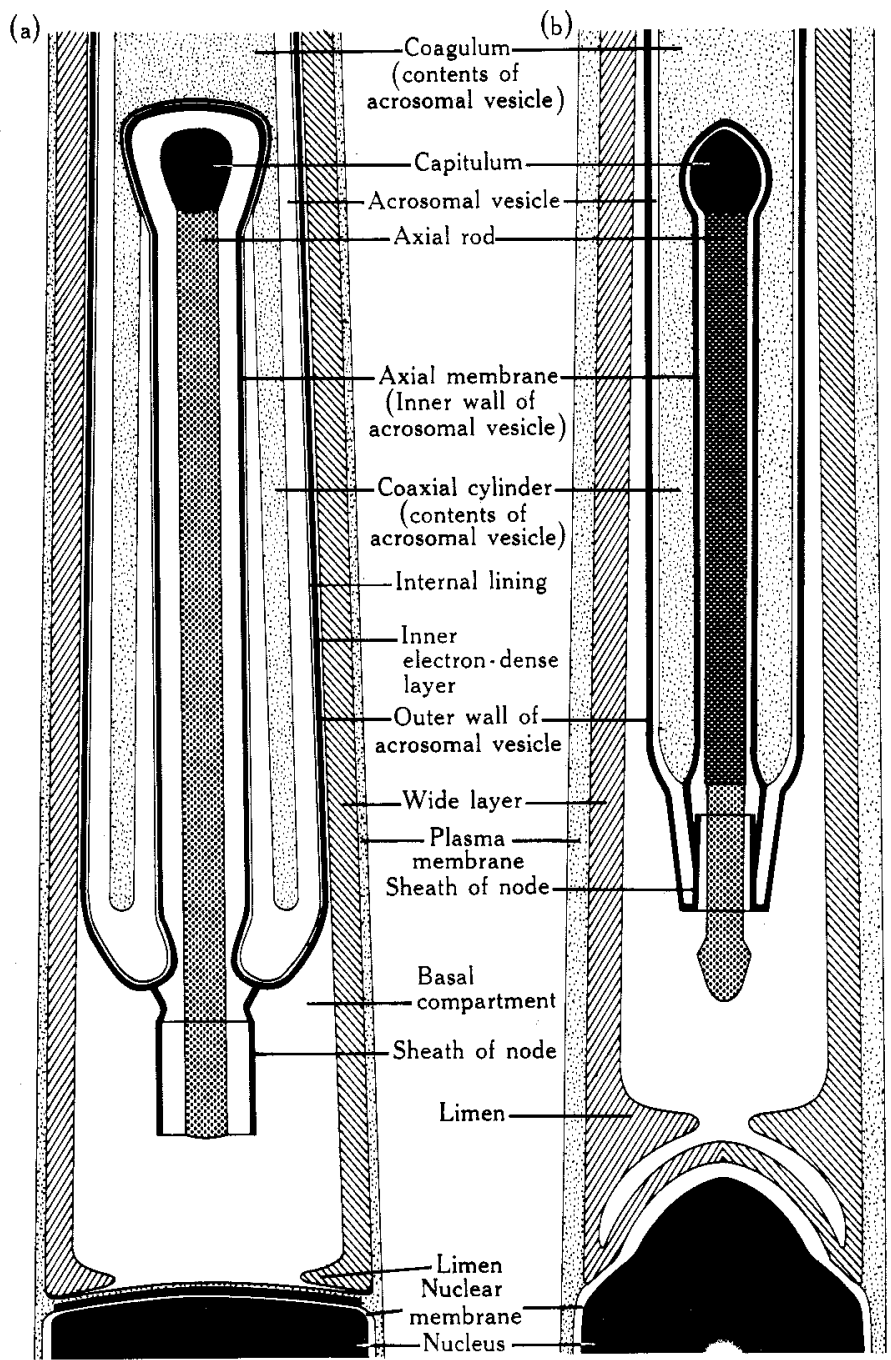

Fig. 2. Comparison and tentative homology of the basal region, including the axial rod, of the acrosome of Lumbricus ( $a$ ) and that of a generalized megascolecid $(b)$. Components in both are approximately to scale. (a) based on micrographs in literature cited. (b) slightly diagrammatized from a camera lucida drawing of the acrosome of Fletcherodrilus unicus but with a shorter axial rod as in Spenceriella.

of the nucleus. In the six megascolecids, on the other hand (e.g. Figs 8, 9, 12 for Fletcherodrilus, Digaster and Amynthas), the proximal end of the nucleus in contact with the acrosome is conspicuously domed, possibly thus giving a stronger and more flexible junction between it and the acrosome. This exemplifies differences between different groups of earthworms which may be utilized for their taxonomic value (see discussion below). 
Further significant differences occur between the megascolecids and Lumbricus in the internal structure of the acrosome, which in both groups appears to be among the most complex reported in the animal kingdom.

\section{The Mature Acrosome}

It will be useful in describing the megascolecid acrosome and comparing it with that of Lumbricus to follow the descriptive sequence for $L$. terrestris adopted by Cameron and Fogal (1963) in their very detailed account. Longitudinal sections of the entire length of the acrosome have been obtained for Fletcherodrilus and for Digaster, from which the following account is chiefly drawn, though comparison is also made with incomplete sections from Cryptodrilus, Spenceriella and Amynthas.

Fig. $2 a$ shows the axial rod and base of the acrosome in Lumbricus slightly diagrammatized from micrographs of Cameron and Fogal, Anderson and Ellis and of Henley; Fig. $2 b$ shows the same region in a megascolecid sperm generalized chiefly from that of Fletcherodrilus, and allows comparison and tentative homology with that of Lumbricus. The acrosomes of Amynthas and its close relative Spenceriella appear to differ considerably from the acrosome of Fletcherodrilus, especially with regard to the configuration of the membranes of the acrosomal vesicle and are under further study.

The acrosome, which is $5-7 \mu \mathrm{m}$ long and located on the flat proximal end of the nucleus in Lumbricus, is only $1.8 \mu \mathrm{m}$ long in Fletcherodrilus, $2.6 \mu \mathrm{m}$ long in Digaster (Figs 8-10), and $1.7 \mu \mathrm{m}$ long in Amynthas sp. (Fig. 12), and is placed on the domed proximal end of the nucleus. Its walls consist of a tube $210 \mathrm{~nm}$ wide (Fletcherodrilus), $280 \mathrm{~nm}$ wide (Digaster) or $260 \mathrm{~nm}$ wide (Amynthas sp.) tapering to a minimal $90 \mathrm{~nm}$ (Fletcherodrilus, Amynthas sp.) or $110 \mathrm{~nm}$ (Digaster) near the discrete rounded tip (350 nm tapering to less than $200 \mathrm{~nm}$ in Lumbricus). Most important for homology with megascolecid spermatozoa, Anderson and Ellis (1968) show the inner electrondense layer turning proximally to invest the axial rod and, contrary to Cameron and Fogal, not passing distally to form the cylinder around the base of the rod.

The layering of the wall of the acrosome in Fletcherodrilus is typical of the six megascolecid species. It agrees with the micrographs for Lumbricus in having two obvious layers (Fig. $2 b$ ): viz. a moderately dense outer layer, $15 \mathrm{~nm}$ wide, which

Fig. 3. A sperm morula of Fletcherodrilus consisting of a central anucleate mass (an) surrounded by young spermatids ( $s$ ) which are connected to it by cytoplasmic bridges. Sections of the nuclei of mature spermatozoa surround the morula. $\times 4400$.

Fig. 4. A portion of an older spermatid of Fletcherodrilus. The nucleus $(n)$ is coarsely granular and mitochondria $(m)$ of the future middle piece are aggregating. The acrosome $(k)$ is developing in a nest of cisternae of the golgi complex $(g) \times 30,800$.

Fig. 5. An anacrosomal spermatid of Fletcherodrilus attached by the zonula collaris $(z)$ to the central anucleate mass $(a n)$ of the morula. The nucleus $(n)$ is elongating and becoming more finely granular. Mitochondria $(m)$ are aggregating in the incipient middle piece and the centriolar complex $(c)$ is visible at the distal end of the flagellum $(f) . \times 19,300$.

Fig. 6. Detail from Fig. 5. Tubules of the manchette $(m a)$ ensheath the nucleus $(n)$ and the mitochondria $(m)$ of the incipient middle piece. Immediately distal to the centriolar complex $(c)$ the dense distal collar (d.co.) is seen at junction of flagellum $(f)$ and middle piece. $\times 44,000$.

Fig. 7. Proximal ends of two older spermatids of Fletcherodrilus. A hollow acrosome ( $a$ ) has been interposed between the nucleus (not shown) and the zonula collaris $(z)$. The manchette ensheathes the acrosome. $\times 46,100$. 


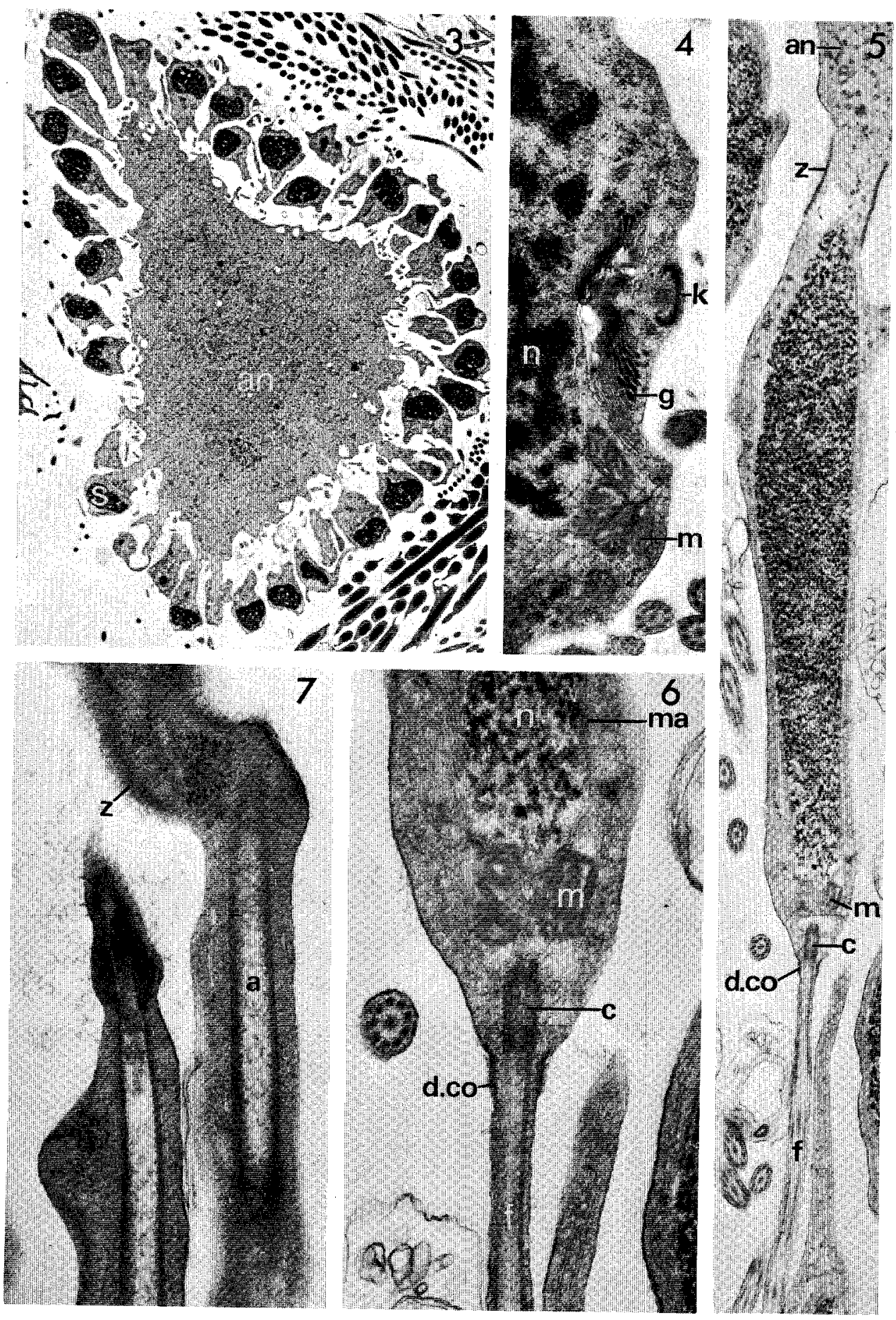




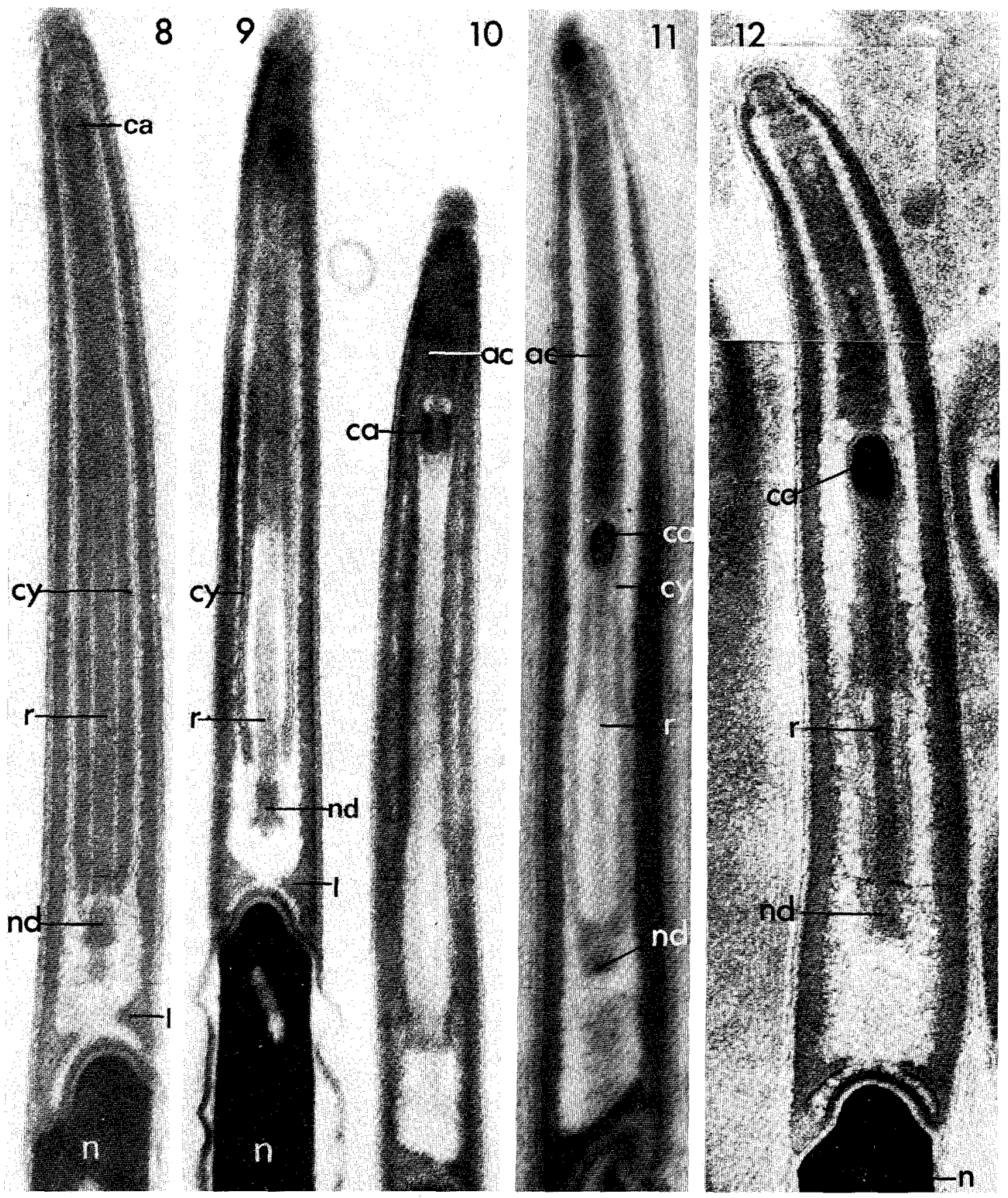

Figs 8-12. Longitudinal sections of mature acrosomes of megascolecids (details in text). 8, Fletcherodrilus, $\times 99,800,9,10$, Digaster, $\times 63,700,11$, Spenceriella, $\times 49,300,12$, Amynthas sp., $\times 97,200.8,10$, from seminal vesicles; 11,12 , from spermathecae. a.c., axial coagulum, $c a$, capitulum, $c y$, coaxial cylinder of granules extending distally from the axial coagulum, 1, flange-like extension (limen) of inner layer of acrosome wall, $n d$, node at base of axial rod, $n$, elongate and lightly condensed, proximally domed nucleus, $r$, axial rod. 

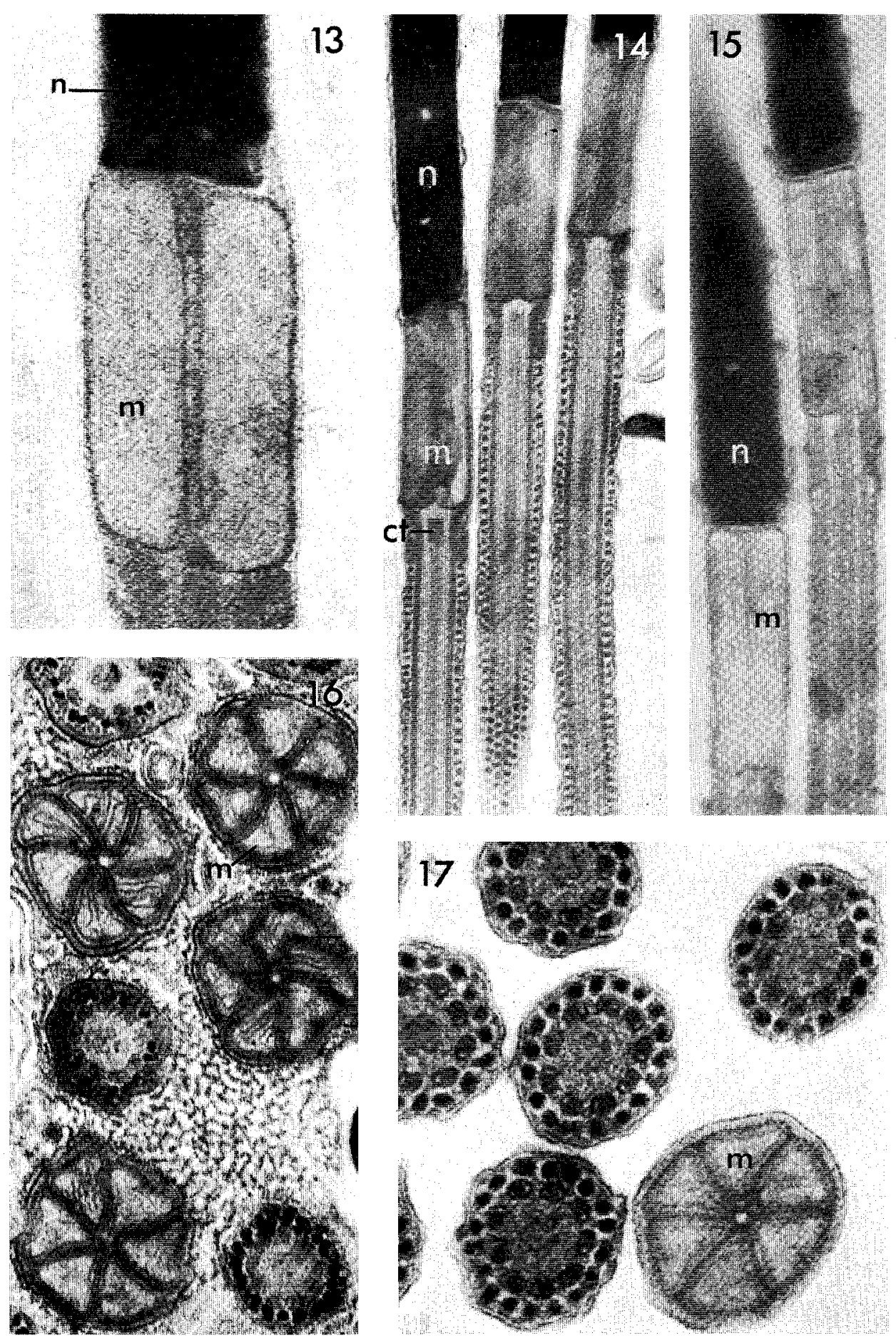

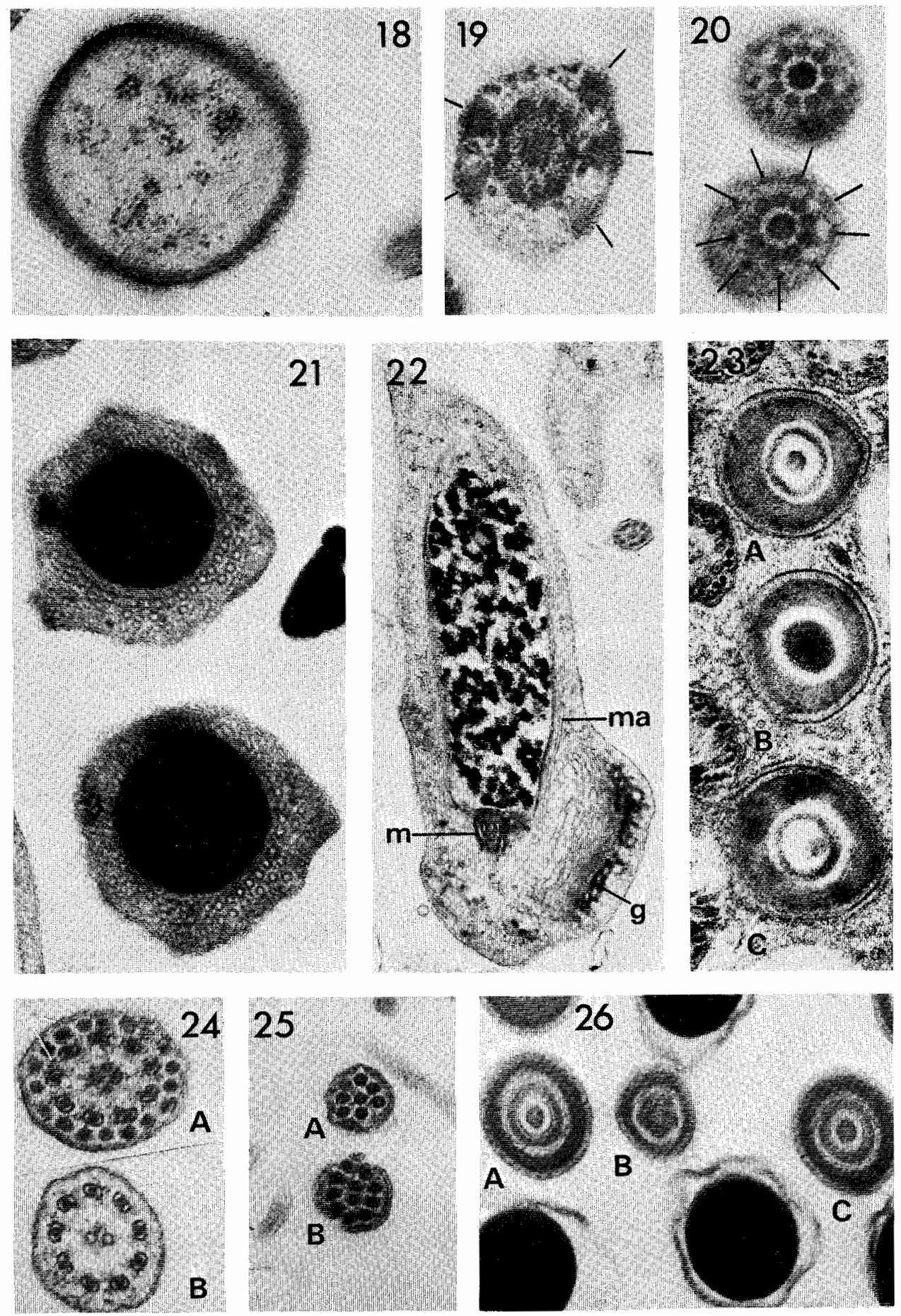

T)

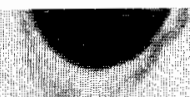

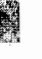


continues somewhat attenuated over the nucleus and middle piece and is identifiable with the plasma membrane described for Lumbricus, and a thicker, very dense layer, $34 \mathrm{~nm}$ wide (Fig. $2 b$, 'wide layer') internal to and adherent to the plasma membrane. Outside the plasma membrane an extremely thin layer is visible in favourable sections and may be a component of the plasma membrane. Unlike the condition in Lumbricus reported by Cameron and Fogal, no layers are visible internal to the two main layers in the external wall but, as demonstrated by Anderson and Ellis for Lumbricus, separate internal sheaths are present. Though these show notable differences in position and degree of development, they can be tentatively homologized with the latter layers in Lumbricus. These layers in sequence in crosssection, as seen in Fig. $26 a$ for Fletcherodrilus, are as follows. First a very thin layer (Fig. $2 b$, 'outer wall of acrosomal vesicle'), of the order of $2 \mathrm{~nm}$ thick, which appears to be the equivalent of the double, innermost membrane of Anderson and Ellis (1968), i.e. the inner electron-dense layer and elusive layer (internal lining) of Cameron and Fogal for Lumbricus. This double layer is shown in the micrograph by Anderson and Ellis to be separated by granular material from the acrosomal wall, so that its distinctly separate nature in megascolecids is only a further development of the

Fig. 13. Middle piece of a spermatozoon in a spermathecal diverticulum of Amynthas sp. $\times 116,000$.

Fig. 14. Three spermatozoa of Fletcherodrilus seminal vesicle in the vicinity of the middle pieces. All show the elongate condensed nucleus $(n)$, the mitochondria $(m)$ of the middle piece and the flagellum with rows of glycogen granules peripheral to the outer tubules of the axoneme. The left sperm shows, within the dense distal collar, the single central tubule $(c t)$ from which the paired central singlets arise. $\times 40,500$.

Fig. 15. Two spermatozoa from a spermatheca of Amynthas diffringens in the vicinity of the middle pieces. $\times 49,500$.

Fig. 16. Transverse sections of four middle pieces of mature spermatozoa in a seminal vesicle of Spenceriella. The radial arrangement of six mitochondria characteristic of megascolecids is clearly seen. Cristae are limited to the juxtaposed walls. $\times 76,300$.

Fig. 17. Transverse section of a middle piece and flagella of spermatozoa from a seminal vesicle of Digaster. The middle piece shows the six radially arranged mitochondria. The flagella show the tetragon arrangement of nine doublets and four central elements (two singlet microtubules +fibres). Glycogen granules surround the doublets. $\times 89,000$.

Fig 18. Transverse section of the zonula collaris of a spermatid in a seminal vesicle of Digaster. The zonula consists of an electron-dense zone around the periphery of the cytoplasmic bridge which is clothed with a nebulous fringe apparently made up of filaments. $\times 45,710$.

Fig. 19. Transverse section, slightly oblique, of the proximal region of a spermatozoal flagellum of Cryptodrilus. Five satellites (arrowed) have a constant asymmetrical arrangement. $\times 85,000$.

Fig. 20. Transverse sections of two late spermatids of Digaster at a level taken to be very shortly distal to the centriole. A single central tubule is surrounded by nine microtubules peripheral to which (arrowed) are nine satellites. $\times 39,100$.

Fig. 21. Transverse sections of two late spermatids of Digaster through the nucleus showing the surrounding microtubules of the manchette. $\times 57,800$.

Fig. 22. Longitudinal section of spermatid of Spenceriella at a stage where the precursor of the middle piece is indicated by location of a mitochondrion $(m)$ at the distal end of the nucleus which is elongating within the manchette $(m a)$. The outer cisternae of the golgi complex $(g)$ are electron-dense. $\times 24,500$.

Fig. 23. Transverse sections of three spermatozoal acrosomes in a seminal vesicle of Spenceriella. $A$ and $C$ are through the axial rod. $B$ is more proximal, through the axial coagulum. $\times 66,000$.

Fig. 24. Transverse sections of two spermatozoal flagella in a seminal vesicle of Fletcherodrilus. $A$, showing the tetragon arrangement with peripheral glycogen granules of the proximal flagellum. $B$, showing the conventional $9+2$ arrangement and absence of glycogen granules further distally. $\times 96,000$.

Fig. 25. As Fig. 24, through the distal end of the flagellum. In $B$ most of the doublets have become singlets; in $B$ only eight singlets persist. $\times 96,000$.

Fig. 26. Transverse sections of three spermatozoal acrosomes in a seminal vesicle of Fletcherodrilus. $A, C$, through the axial rod. $B$, apparently through the capitulum. Sections of nuclei are also seen. $\times 67,000$. 
lumbricid condition. Secondly, internal to this outer wall of the acrosomal vesicle is a very electron-dense broad layer (Figs $2 a, 2 b$, 'coaxial cylinder') $15 \mathrm{~nm}$ thick, which is here considered the equivalent of the layer described by Cameron and Fogal for Lumbricus, extending from the finely granular material anterior to the axial rod (Fig. 2, 'coagulum') as a (coaxial) cylinder around the rod (Fletcherodrilus; Digaster; Figs $8,9)$. Coagulum and coaxial cylinder constitute the contents of the acrosomal vesicle.

Figs 23 and 26, for Spenceriella and Fletcherodrilus, show transverse sections of the acrosome at the level $(A, C$, in both) of the axial rod and coaxial cylinder and $(B)$ at the more proximal level of the axial coagulum. Thirdly, internal to the coaxial cylinder is a further delicate membrane of the order of $2 \mathrm{~nm}$ thick (Fig. 26a; Fletcherodrilus) which appears to be the equivalent of the membrane noted for Lumbricus (Figs $2 a, 2 b$, 'axial membrane') surrounding the axial rod, though in Lumbricus it has a thickness of $16 \mathrm{~nm}$. This is the inner wall of the acrosomal vesicle. In Fletcherodrilus and in Digaster (Figs 8,9) the two delicate membranes (outer and inner walls of the acrosomal vesicle) have been traced distal to the coaxial cylinder, which it appears their function to contain, and unite with the node (Figs $2 b, 8-10$ ) at the base of the axial rod. Although on initial consideration the existence of a basal node and the contiguity of the outer and inner membranes with this appear to distinguish megascolecid sperm from the sperm of Lumbricus, it will be shown in discussion of the base of the acrosome that a structure comparable with the basal node exists in Lumbricus and that two membranes bounding the poorly developed coaxial cylinder become contiguous at it (Fig. $2 a$ ).

\section{The Axial Rod and the Base of the Acrosome}

Cameron and Fogal (1963) report that in Lumbricus part of the base of the acrosome forms a compartment about $360 \mathrm{~nm}$ long, separated from the main body of the acrosome by a transverse partition which is penetrated by the base of the axial rod. The micrograph of Anderson and Ellis reveals that, on the contrary, both inner layers lining the acrosome wall return proximally to ensheath the axial rod and that the cylinder (Fig. 2, 'sheath of node') around the base of the rod is a separate structure, though connected with the transverse partition. The micrograph also reveals that the transverse partition may be strongly convex distalwards, around the axial rod, as depicted here (Fig. 2a). This, combined with the continuation of the granular material from the basal compartment proximally between the wide layer of the acrosome wall and the double membrane, greatly reduces the discreteness of the basal compartment. The existence of a transverse septum and basal compartment is thus a somewhat illusory difference between the lumbricid and megascolecid acrosomes; though not entirely spurious. In Lumbricus what has here been termed the coaxial cylinder lies in a cavity within the reflexed double membrane. This is the cavity of the acrosomal vesicle, of which the double membrane forms the inner and outer walls.

The axial rod of Lumbricus is almost $1 \mu \mathrm{m}$ long and $55 \mathrm{~nm}$ wide, with the spherical, relatively electron-dense tip $90 \mathrm{~nm}$ in diameter. In the megascolecids, although the acrosome is less than one-third of the length of that in Lumbricus, the axial rod is relatively longer. Lengths, general width and width of the terminal dilation respectively are approximately: $1 \cdot 3 \mu \mathrm{m}, 30 \mathrm{~nm}, 44 \mathrm{~nm}$ (Fletcherodrilus); $1 \cdot 7 \mu \mathrm{m}, 56$ $\mathrm{nm}, 73 \mathrm{~nm}$ (Digaster); $1.4 \mu \mathrm{m}, 42 \mathrm{~nm}, 130 \mathrm{~nm}$ (Spenceriella); $0.8 \mu \mathrm{m}, 30 \mathrm{~nm}, 80 \mathrm{~nm}$ (Amynthas sp.); and 1.0 $\mu \mathrm{m}, 37 \mathrm{~nm}, 50 \mathrm{~nm}$ (Amynthas diffringens); measured in each case for a single acrosome. Striking differences from the acrosome of Lumbricus are 
the less discrete basal juxtanuclear compartment, the dome-shaped floor of the acrosomal vesicle, a reflection of the convex form of the proximal end of the nucleus, and the invariable presence of an inward projection (Fig. 2 $b$. 'limen') of the wide layer of the acrosomal wall, very near the floor of the vesicle, as a flange with central orifice. The limen, though perforate, conforms with the floor of the acrosome in its domed form. There is a suggestion in lumbricid micrographs of a similar though lesser flange-like inward projection of the corresponding layer of the acrosome wall near the floor of the basal compartment. The equivalent of the basal compartment in megascolecids contains diffuse, coarsely granular, moderately electron-dense material. The structure of the basal node of the axial rod in megascolecid sperm has not been precisely determined but an attempt at elucidation is made in Fig. $2 b$. In the micrographs from Fletcherodrilus (Fig. 8) and Digaster (Fig. 9) it is seen that the node is penetrated by the axial rod, which projects for a short distance distal to it. The node peripheral to its axial rod is tentatively interpreted as a short ensheathing cylinder ('sheath of node') equivalent to that in Lumbricus. The inner and outer walls of the acrosomal vesicle connect to it more distally than do their apparent homologues in Lumbricus.

\section{Tip of the Acrosome}

Fig. 8, for Fletcherodrilus, shows one of the more favourable preparations of the acrosome tip. Little of its structure can be resolved with certainty, but constant features are visible in the Fletcherodrilus preparations which are depicted in Fig. 1a. The acrosome tip of Amynthas sp. is shown in Fig. 12.

Cameron and Fogal describe and illustrate external papillation of the plasma membrane near the tip of the lumbricid acrosome. The proximal third and more of the acrosome wall in Fletcherodrilus has a scantily hirsute appearance and some of the projecting filaments appear to include extensions of the wide layer underlying the plasma membrane, as well as of the latter. No workers to date appear to have reported for lumbricids the filopod-like, very slender filaments which radiate far out from the domed top of the acrosome of Fletcherodrilus. Their nature is uncertain but they might represent extruded 'secretion' (perhaps as a response to fixation) or structural elements of the acrosome concerned with establishing adhesion to the eggs.

\section{The Middle Piece}

Middle pieces have been observed in longitudinal and transverse section for the six megascolecid species under investigation. Longitudinal sections are illustrated for Amynthas sp. (Fig. 13), A. diffringens (Fig. 15), and Fletcherodrilus (Fig. 14) and transverse sections for Spenceriella (Fig. 16) and Digaster (Fig. 17). After the precursory stage shown in Figs 5 and 6 , the six mitochondria come together around a slender clear cylindroid axis, their juxtaposed faces (here termed septa) radiating from this like the spokes of a wheel. Each mitochondrion in transverse section in the megascolecid species shows one or two cristae arising from the juxtaposed walls, but never from the external walls, and crossing most of the lumen of the mitochondrion (Fig. 16). The definitive mitochondria of the middle piece in Lumbricus have a similar appearance (Anderson et al. 1967; Lanzavecchia and Donin 1972). There a progressive loss of microtubules of the manchette and of cytoplasm from the incipient middle piece of the spermatid occurs until, in the mature middle piece, only a single layer, the plasma membrane, persists outside the mitochondria. 


\section{The Sperm-tail (Flagellum)}

Henley (1973), to whom we owe most of our knowledge of the axoneme of the earthworm (lumbricid) spermatozoon, states that it has the usual $9+2$ pattern of microtubules modified in various ways. Using negative staining she demonstrated that glycogen granules peripheral to the nine doublets have a physical attachment to the doublets. Similar granules are present in all mature megascolid sperm investigated, distal to the dense collar (Figs 14, 17, 24A) extending far down the flagellum. There is no reason to doubt that these are glycogen, as shown by Anderson and Personne (1970) for the axoneme of Lumbricus terrestris. In Digaster, as noted by Anderson et al. for Lumbricus, immediately distal to the centriole the axoneme has a single large central microtubule. Around this (Fig. 20, Digaster) are nine tubules which appear to be doublets, though it is not certain that they are not triplets as observed by Anderson et al. for Lumbricus. It is not known whether the nine satellites demonstrated in this region in Digaster are general in megascolecids. The single axial microtubule gives rise, as noted by Anderson et al., to the two more distal central tubules but, as noted by Henley for Lumbricus, two additional elements are found in the axonemes, smaller than the two singlets of the main part, differing in being solid and forming with them a tetragon configuration (Fig. 17, Digaster; Fig. 24A, Fletcherodrilus).

A study of the megascolecid axonemes reveals that the tetragon arrangement does not continue throughout the flagellum but that it is replaced further distally by the normal $9+2$ arrangment (Fig. 24B). In Fletcherodrilus one of the additional, solid, fibres may disappear before the other, and the glycogen granules may reduce in size in this transitional zone and persist only a short distance into the $9+2$ region. At the distal end of the axoneme the peripheral doublets gradually, but not simultaneously, become singlets (Fig. 25B). Terminally no central pairing is observable, and eight (Fig. 25 A) and successively less of the singlets remain.

It is of great interest for taxonomy that differences exist between species in the axoneme as in the acrosome and in other features of the sperm. Thus, in Cryptodrilus (Fig. 19), very numerous cross-sections have been obtained in which five large satellites are present outside the nine peripheral tubules. The large number of these sections and the presence in them of glycogen granules suggest that they are well distal to the centriole. A remarkable feature of the satellites is their asymmetrical disposition, with three on one side and two close together on the other side, well separated from the three.

\section{The Mature Spermatozoon}

In the mature sperm the manchette and cytoplasm peripheral to it have been eliminated. The earthworm spermatozoon shows extreme elongation, relative to width, of acrosome, nucleus and middle piece, though the middle piece is short relative to that in many other groups. In the single spermatozoon of Fletcherodrilus for which a complete longitudinal section from acrosome to tail has been obtained, lengths and widths respectively are: acrosome 1.76 by $0.21 \mu \mathrm{m}$; nucleus 8.69 by $0.21 \mu \mathrm{m}$, and middle piece 0.64 by $0.20 \mu \mathrm{m}$. The total length of the head (acrosome plus nucleus) is $10.45 \mu \mathrm{m}$, compared with $23-26 \mu \mathrm{m}$ and a middle piece length of $2 \mu \mathrm{m}$ given by Chatton and Tuzet (1941) for Lumbricus terrestris. The structure of the various components and some aspects of their development have been discussed earlier, and 
more detailed measurements provided, and it will suffice to summarize the structure of a generalized megascolecid spermatozoon in Fig. 1.

\section{Discussion}

Although the megascolecid spermatozoon shows some significant and, as far as has been demonstrated, constant differences from the spermatozoon of Lumbricus, careful analysis has revealed general spermatozoal homology between their respective families. Nevertheless the differences are such that on present evidence there would be no difficulty in determining whether a spermatozoon were lumbricid or megascolecid. Furthermore, differences of detail exist among the six megascolecids which promise to be of great value to the specialist taxonomist in establishing classifications, though it is questionable that they would be of value in routine taxonomic work. It has yet to be established whether differences observed are specific criteria or pertain to supraspecific groupings such as the genus. Variation of spermatozoal ultrastructure in the Megascolecidae and especially in the Lumbricidae, in which only that of Lumbricus terrestris has been examined, requires much further investigation, and it is expected that spermatozoal morphology may aid in selecting the best of the several conflicting internal classifications of the Megascolecidae (Gates 1959; Lee 1959; Jamieson 1971). The sperm of Limnodrilus and Tubifex have been shown by Ferraguti and Lanzavecchia (1971) to differ from those of lumbricids (and megascolecids) in having spiral nuclei and only two mitochondria. Five or six mitochondria (individual variation) are present in the middle pieces of other tubificids studied in this laboratory, however, while nuclei are straight or spiral.

In a wider taxonomic context, the structure of the oligochaete spermatozoon approaches that of, at least, the leech Platybdella annarrichae (fide Fränzen 1956), the oligochaetes and leeches being subclasses of the class Clitellata (see Brinkhurst and Jamieson 1971). Those of the remaining annelid classes, the Polychaeta and Archiannelida, are, as was predictable, very different from those of the clitellates. In the polychaetes they are usually of the 'primitive type' (Fränzen 1956, 1970) with four spherical mitochondria in the middle piece, subspheroidal nucleus and low, cap-like acrosome (Fränzen 1956, 1970). The ultrastructure of this type of polychaete spermatozoon has been lucidly demonstrated by Graebner and Kryvi (1973) for Sabella penicillum. In the Archiannelida, Polygordius has retained the primitive type. More or less profound modifications of the primitive form occur in the sperm of a small proportion of the many polychaete species, and the remainder of the few archiannelid species, which have been investigated, but none approaching the oligochaete spermatozoon in morphology is known. The utility of spermatozoal morphology for taxonomy and phylogeny is demonstrated by capitellid polychaetes, the general morphology of which has led to the view that they have an especially close relationship with oligochaetes. The spermatozoa of the two groups give no support to this contention (Fränzen 1956).

\section{Acknowledgments}

Mr J. Hardy, Electron Microscope Section, University of Queensland, is thanked for his invaluable aid in the preparation of the micrographs, and $\mathrm{Mr} \mathrm{J}$. Casey and Mrs L. Daddow for assistance. This work was made possible by an Australian Research Grants Committee grant. 


\section{References}

Anderson, W. A., and Ellis, R. A. (1968). Acrosome morphogenesis in Lumbricus terrestris. Z. Zellforsch. Mikrosk. Anat. 85, 398-407.

Anderson, W. A., and Personne, P. (1970). The localization of glycogen in the spermatozoa of various invertebrate and vertebrate species. $J$. Cell Biol. 44, 29-51.

Anderson, W. A., Weissman, A., and Ellis, R. A. (1967). Cytodifferentiation during spermiogenesis in Lumbricus terrestris. J. Cell Biol. 32, 11-26.

Baird, W. (1869). Description of a new species of earthworm (Megascolex diffringens) found in North Wales. Proc. Zool. Soc. Lond. 1869, 40-43.

Boardman, W. (1932). Some earthworms from Queensland. Mem. Queensl. Mus. 10 (2), 125-30.

Brinkhurst, R. O., and Jamieson, B. G. M. (1971). 'The Aquatic Oligochaeta of the World.' (Oliver and Boyd: Edinburgh, London, Toronto.)

Bugnion, E., and Popoff, N. (1905). La spermatogénèse du Lombric terrestre. Arch. Zool. Exp. 3, 339-89.

Cameron, M. L., and Fogal, W. H. (1963). The development and structure of the acrosome in the sperm of Lumbricus terrestris L. Can. J. Zool. 41, 753-61.

Chatton, E., and Tuzet, O. (1941). Sur quelque faits nouveaux de la spermatogénèse du Lumbricus terrestris. C. R. Hebd. Seances Acad. Sci. 213, 373-6.

Fawcett, D. W. (1966). 'An Atlas of Fine Structure: the Cell; its Organelles and Inclusions.' (Saunders: Philadelphia.)

Ferraguti, M., and Lanzavecchia, G. (1971). Morphogenetic effects of microtubules. 1. Spermiogenesis in Annelida Tubificidae. J. Submicrosc. Cytol, 3, 121-37.

Fränzen, A. (1956). On spermatogenesis, morphology of the spermatozoon and biology of fertilization among invertebrates. Zool. Bidr. Upps. 31, 355-482.

Fränzen, A. (1970). In 'Comparative Spermatology'. (Ed. B. Baccetti.) (Academic Press: London.)

Gatenby, J. B., and Dalton, A. J. (1959). Spermiogenesis in Lumbricus herculeus. An electron microscope study. J. Biophys. Biochem. Cytol. 6, 45-52.

Gates, G. E. (1959). On a taxonomic puzzle and the classification of the earthworms. Bull. Mus. Comp. Zool. Harv. Coll. 121, (6), 229-61.

Graebner, I., and Kryvi, H. (1973). Spermiogenesis and mature sperm of Sabella penicillum (Polychaeta). An electron microscopical investigation. Norw. J. Zool. 21, 211-66.

Henley, C. (1973). Ultrastructure of the spermatozoon of the earthworm as revealed by negative staining. J. Morphol. 140, 197-214.

Jamieson, B. G. M. (1971). A review of the megascolecoid earthworm genera (Oligochaeta) of Australia. Part $\mathrm{I}$ - Reclassification and checklist of the megascolecoid genera of the World. Proc. R. Soc. Queensl. 82 (6), 75-86.

Lanzavecchia, G., and Donin, C. L. L. (1972). Morphogenetic effects of microtubules II. Spermiogenesis in Lumbricus terrestris, J. Submicrosc. Cytol. 4, 247-60.

Lee, K. E. (1959). The earthworm fauna of New Zealand. Bull. N.Z. Dep. Sci. Ind. Res. No. 130. Michaelsen, W. (1891). Oligochaeten des Naturhistorischen Museums in Hamburg. IV. Jahrb. Hamb. Wiss. Anst. 8, 299-350.

Reger, J. F. (1967). A study on the fine structure of developing spermatozoa from the oligochaete, Enchytraeus albidus. Z. Zellforsch. Mikrosk. Anat. 82, 257-69.

Walsh, M. P. (1954). Spermatogenesis of Lumbricus terrestris L. Trans. Am. Microsc. Soc. 73, 59-65. 